n.tronos

glyndŵn

Glyndŵr University

Glyndŵr University Research Online

Computing

Computer Science

$1-1-1988$

\title{
A Simple Algorithm for Sidelobe Cancellation in a Partially Adaptive Linear Array
}

IEl-Azhary

MS. Afifi

Peter S. Excell

Glyndwr University, p.excell@glyndwr.ac.uk

Follow this and additional works at: http://epubs.glyndwr.ac.uk/cair

Part of the Computer Sciences Commons

\section{Recommended Citation}

El-Azhary, I., Afifi, M. S., \& Excell, P.S. (1988) 'A Simple Algorithm for Sidelobe Cancellation in a Partially Adaptive Linear Array'. IEEE Transactions on Antennas and Propagation, 36(10), 1482-1486

This Article is brought to you for free and open access by the Computer Science at Glyndŵr University Research Online. It has been accepted for inclusion in Computing by an authorized administrator of Glyndŵr University Research Online. For more information, please contact

d.jepson@glyndwr.ac.uk. 


\title{
A Simple Algorithm for Sidelobe Cancellation in a Partially Adaptive Linear Array
}

\begin{abstract}
The far-field lobes of the edge elements of a uniformly excited linear array are nearly equal in width to the sidelobes of the array itself, and hence the edge elements are ideal for cancellation of specific sidelobes of the pattern. This supports the concept of partial adaptivity using only the edge elements. This technique is suitable for real-time implementation because only the edge elements require direct control whereas the inner elements are controlled by PROM's. Other advantages are that the nulls produced specifically by control of the edge elements are deep and wide. Also the main beam gain is not much affected by the sidelobe cancellation.
\end{abstract}

\section{Keywords}

partial adaptivity, edge elements, real-time implementation, PROM’s

\section{Disciplines \\ Computer Sciences}

\section{Comments}

(C)1988 IEEE. Reprinted, with permission, from "El-Azhary, I., Afifi, M. S., \& Excell, P.S. Journal of IEEE Transactions on Antennas and Propagation, Vol.36, No.10 pp.1482-1486 Oct 1988 . Personal use of this material is permitted. However, permission to reprint/republish this material for advertising or promotional purposes or for creating new collective works for resale or redistribution to servers or lists, or to reuse any copyrighted component of this work in other works must be obtained from the IEEE. This material is presented to ensure timely dissemination of scholarly and technical work. Copyright and all rights therein are retained by authors or by other copyright holders. All persons copying this information are expected to adhere to the terms and constraints invoked by each author's copyright. In most cases, these works may not be reposted without the explicit permission of the copyright holder. The definitive version is available at http://ieeexplore.ieee.org 
with the $0.1 \mathrm{~mm}$ claimed for our method. A further advantage of this technique is the fact that the basic observations, the taking of several photographs, requires relatively little time, so that the results will be reasonably free of thermal changes. Further, the observations can be made at a variety of antenna orientations, so that structural distortions could be observed. A drawback is the time required for the analysis: the photographs need to be digitized, and the data then processed before a set of panel adjustment instructions can be issued. A recent demonstration of the technique involving 110 points and six photographs required 50-min measuring time, and 20-min reduction time. The number of points on a large radio telescope is more likely in the vicinity of 1000 . Fraser [3] quotes $18 \mathrm{~h}$ for the time to scan the images of a 34-m antenna.

Holography: This technique [3] has several advantages over the alternative schemes: it provides a true measure of the performance of the reflector/subreflector system, since it measures the phase variations across the outgoing wavefront. However, this method has a cost: high signal-to-noise is required, which in general translates to a requirement for long integrations and hence the operation may take several hours. Furthermore, interpretation in case of dual-reflector antennas is not straightforward.

\section{CONCLUSION}

In this communication we have described a survey method which has allowed us to adjust the reflector surface of large radio telescopes to high accuracy: we claim an achieved overall setting accuracy of better than $0.25 \mathrm{~mm}$.

\section{REFERENCES}

[1] R. H. Frater, "The Australia telescope-The radio astronomer's highway to the future," Proc. Astron. Soc. Aust., vol. 5, p. 440, 1984.

[2] C. S. Fraser, "Microwave antenna measurement," Photogramm. Eng. Remote Sensing, no. 10, p. 1627, 1986.

[3] P. F. Scott and M. Ryle, "A rapid method for measuring the figure of a radio telescopt reflector," Mon. Not. R. Astron. Soc., vol. 178, p. $539,1977$.

\section{A Simple Algorithm for Sidelobe Cancellation in a Partially Adaptive Linear Array}

ISMAIL EL-AZHARY, MEMBER, IEEE, MOSTAFA S. AFIFI, SENIOR MEMBER, IEEE, AND PETER S. EXCELL, SENIOR MEMBER, IEEE

Abstract-The far-field lobes of the edge elements of a uniformly excited linear array are nearly equal in width to the sidelobes of the array itself, and hence the edge elements are ideal for cancellation of specific sidelobes of the pattern. This supports the concept of partial adaptivity using only the edge elements. This technique is suitable for real-time implementation because only the edge elements require direct control whereas the inner elements are controlled by PROM's. Other advantages

Manuscript received October 22, 1986; revised September 3, 1987.

El-Azhary and M. S. Afifi are with the Department of Electrica Engineering, King Saud University, P.O. Box 800 Riyadh 11421, Saudi Arabia.

P. S. Excell is with the School of Electrical and Electronic Engineering, University of Bradford, Bradford BD7 1DP, U.K.

IEEE Log Number 8822581 . are that the nulls produced specifically by control of the edge elements are deep and wide. Also the main beam gain is not much affected by the sidelobe cancellation.

\section{INTRODUCTION}

An important class of adaptive antennas is the class of sidelobe cancellers (with possible beam maximization requirement) that were among the first applications of adaptive arrays [1]. Many approaches are possible, depending on the adaptivity algorithm utilized. Most of these algorithms are time-consuming and are difficult to implement in real-time because they involve complex iterations and matrix operations [2]-[5].

Sometimes only few elements of the array are controllable, e.g., the Howells sidelobe canceller [6], and in this case the array is said to be partially adaptive. Full adaptivity (control of all elements) could be prohibitively expensive in many applications, especially when the array contains a large number of elements [7], and may raise reliability problems due to the large number of components required and the complexity of the controlling processor.

In this communication, a simple algorithm for cancelling specific sidelobes, using edge elements alone, is presented.

\section{The Relation Between the Sidelobes and the Pattern of} THE EDGE ELEMENTS

Consider a linear array with $\mathrm{N}+1$ elements separated by equal intervals of size $h$. Assume a uniform excitation function such that $w(n)=1$ for all elements. In this case, it is well known that the modulus of the array factor $f_{a}(\theta)$ is given by

$$
f_{a}(\theta)=\frac{\sin \left(\frac{N+1}{2} k h \sin \theta\right)}{\sin \left(\frac{k h}{2} \sin \theta\right)} .
$$

Fig. 1 shows the pattern due to the edge elements alone (the solid curve) together with the pattern of a uniformly excited 11-element array (the dotted curve). It is clear that the pattern due to the edge elements, given by

$$
\begin{aligned}
f & =e^{j k h(N / 2) \sin \theta}+e^{-j k h(N / 2) \sin \theta} \\
& =2 \cos \left[\frac{k h N}{2} \sin \theta\right]
\end{aligned}
$$

forms a cosine pattern with almost the same sidelobe structure and periodicity as the sidelobes of the complete array pattern.

\section{An Algorithm for Cancelling SPECific Sidelobes}

A simple algorithm is now presented to cancel any selected sidelobe by making use of this property.

Let there be an interfering signal coming from the direction $\theta_{\text {int }}$. Instead of creating a null in this direction only, it is more convenient to cancel the whole sidelobe which contains the angle $\theta_{\text {int }}$. A simple search algorithm can be used in determining which sidelobe contains the angle $\theta_{\text {int }}$.

In order to cancel the whole sidelobe, it is first necessary to determine the angle $\theta_{m}$ of its center. Then by adding a cancellation signal with conjugated phase shifts to the first and the last elements, respectively, a superimposed cosine pattern is created which can be shifted left or right in order that one of its peaks coincides with $\theta_{m}$. Now, by scaling the amplitude of the cosine pattern by a factor $C$, 


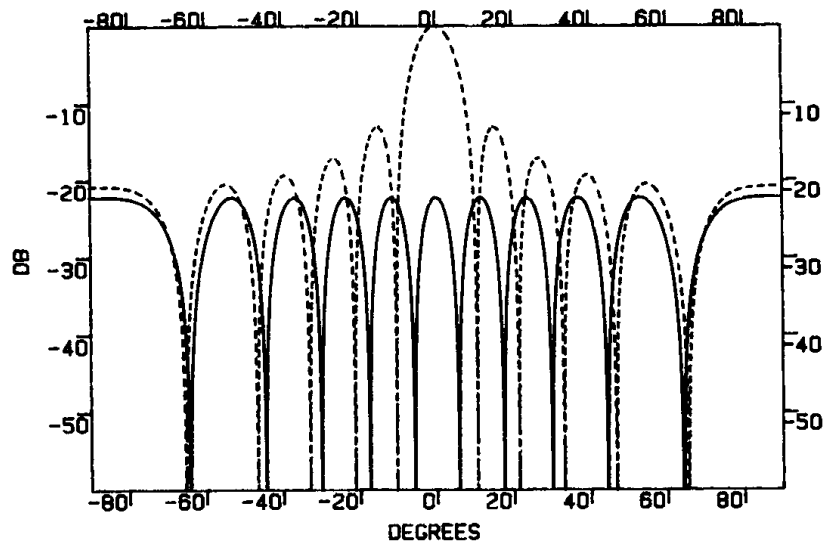

Fig. 1. The pattern due to the edge elements alone (solid curve), compared with the pattern of an equispaced 11-element uniformly excited linear array (dotted curve).

say, so that it is equal in magnitude to and in antiphase with the array pattern at $\theta_{m}$, the sidelobe in question will be cancelled.

The arithmetic involved in the above procedure is straightforward, as follows

1) The maxima of the sidelobes occur at angles $\theta_{m}$, where

$$
\sin \theta_{m}= \pm \frac{(2 m+1)}{N+1}
$$

where $N+1$ is the total number of elements in the array, $m=1,2$, $\cdots, N / 2$ is the index of the sidelobe to be cancelled.

2) Knowing the angle $\theta_{m}$ and assuming a $\lambda / 2$ spacing between elements (i.e., $k h=\pi$ ) and assuming an odd number of elements, the corresponding maxima $f_{m}$ can be computed from (1)

$$
\left|f_{m}\right|=\frac{1}{\left|\sin \left[\frac{\pi}{2} \frac{(2 m+1)}{(N+1)}\right]\right|}
$$

3) Apply conjugated phase shifts $\delta$ and $-\delta$ to the cancellation signal of amplitude $C$ fed to the edge elements. The cancellation pattern is then given by

$$
\begin{gathered}
f_{c}\left(\theta_{m}\right)=C e^{j((\pi / 2) N(2 m+1) /(N+1)+\delta)+C e^{-j((\pi / 2) N(2 m+1) /(N+1)+\delta)}} \\
2 \cos \left[\frac{\pi}{2} \frac{N}{N+1}(2 m+1)+\delta\right]
\end{gathered}
$$

4) At $\theta_{m}$,

$$
f_{c}\left(\theta_{m}\right)=-f_{m}
$$

If the peak of the cosine pattern is registered with the peak of the sidelobe in question, the value of the cosine term in (5) is unity and therefore,

$$
|C|=\left|f_{c}\left(\theta_{m}\right)\right| / 2=\left|f_{m}\right| / 2
$$

and

$$
\delta=-\left[\pi+\frac{\pi}{2} N \frac{(2 m+1)}{(N+1)}\right] .
$$

5) Knowing $C$ and $\delta$, the excitation of the edge elements that would produce a pattern whose $m$ th sidelobe is cancelled, can be computed. The required excitation $w_{\text {new }}(n) e^{j \phi n}$ is simply the superposition of the

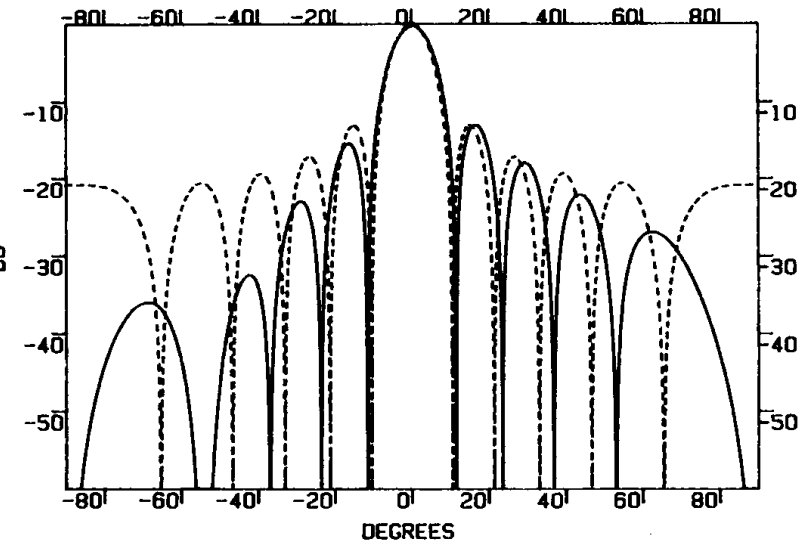

Fig. 2. The effect of cancelling the fourth sidelobe (solid curve) compared with the original pattern of the uniformly excited array (dotted curve).

new cancellation signal and the original signal.

$$
\begin{aligned}
\left|w_{\text {new }}(0)\right| & =\left|w_{\text {new }}(N)\right| \\
& =\left\{\left(1-(-1)^{m} C \cos \delta\right)^{2}+C^{2} \sin ^{2} \delta\right\}^{1 / 2}
\end{aligned}
$$

and

$$
\phi_{N}=\arctan \left\{\frac{C \sin \delta}{1-(-1)^{m} C \cos \delta}\right\}
$$

and

$$
\phi_{0}=-\phi_{N}
$$

It is clear that interchanging the signs of $\phi_{N}$ and $\phi_{0}$ results in cancelling the sidelobe at $-\theta_{m}$.

Fig. 2 shows the cancellation of the fourth sidelobe for a uniformly excited 11-element array.

\section{CONCLUSION}

It is clear that the main lobe gain is not greatly affected since only two elements of the whole array are used. Additionally, the individual nulls produced are wide enough to accomodate frequency fluctuations, usually overcome in conventional techniques by placing two adjacent nulls in the radiation pattern [8].

The algorithm for sidelobe cancellation presented in this communication required full control of both the amplitude and phase of the feeds to the edge elements. However, the most important advantage of the algorithm is that it is suitable for real-time implementation, since, knowing the excitation function and the number of elements of the array, the values of $w_{\text {new }}$ and $\phi$ can be computed and stored in look-up tables and these values are less than half of the total number of elements of the array. This means, once the sidelobe to be cancelled is determined, its $w_{\text {new }}$ and $\phi$ are issued immediately without delays due to matrix operations or iterations. For a nonsteerable array this gives a great reduction in the number of RF devices. For steerable arrays all elements must have active devices in the feeds, but the algorithm is simpler than that for full adaptivity because it involves direct control for the edge elements alone whereas the interior elements may be controlled indirectly using PROM's.

\section{REFERENCES}

[1] W. F. Gabriel, "Adaptive processing antenna systems," IEEE Antennas Propagat. Soc. Newsletter, vol. 25, no. 5, pp. 5-11, 1983.

[2] IEEE Trans. Antennas Propagat., Special issue on active and adaptive antennas, vol. AP-12, no. 2, 1964. 
[3] IEEE Trans. Antennas Propagat., Special issue on adaptive antennas, vol. AP-24, no. 2, 1976.

[4] Proc. Inst. Elec. Eng., Special issue on adaptive arrays, vol. 130, pts. $\mathrm{F}$ and $\mathrm{H}$, no. 1,1983 .

[5] IEEE Trans. Antennas Propagat., Special issue on adaptive processing antenna systems, vol. AP-34, no. 3, 1986.

[6] P. W. Howells, "Exploration in fixed and adaptive resolution at GE and SURC," IEEE Trans. Antennas Propagat., vol. AP-24, no. 3, pp. $575-584,1976$.

[7] D. J. Chapman, "Partial adaptivity for the large array," IEEE Antennas Propagat., vol. AP-24, no. 5, pp. 685-696, 1976.

[8] P. Owen and J. C. Mason, "The use of linear programming in the design of antenna patterns with prescrived nulls and other constraints," COMPEL, vol. 3, no. 4, pp. 201-215, Dec. 1984

\section{A Note on Hybrid Finite Element Method for Solving Scattering Problems}

\section{JIAN-MING JIN AND VALDIS V. LIEPA, MEMBER, IEEE}

\begin{abstract}
The hybrid finite element formulation is modified so that it results in a sparse banded symmetric matrix. This modification substantially improves the computational efficiency and enhances the capability of the method, which is demonstrated by numerical examples. A comparison with other numerical techniques is presented.
\end{abstract}

\section{INTRODUCTION}

The hybrid finite element method (HFEM) was first introduced by Silvester and Hsieh [1] and McDonald and Wexler [2] in the early 1970 's, and has been further improved and applied to solving various two-dimensional unbounded field problems, e.g., [3]-[9]. The method expresses the exterior fields using an eigenfunction series or an integral involving a Green's function. Such expressions are imposed on the variational equation or, more conveniently, on the matrix equation derived using the finite element method for the interior fields. As a result, HFEM produces a system matrix having a nonuniform block submatrix structure. Such a matrix, as pointed out by Mei and Morgan [10], [11], is not easily adaptable to banded matrix algorithms, and hence is numerically inefficient to solve using direct or iterative methods. As a consequence, HFEM in such a form is less efficient than the unimoment method [10]-[12] and its modification-the so-called hybrid finite element-extended boundary condition method (hybrid FEM-EBCM) [13], mainly because the unimoment method and the hybrid FEM-EBCM can take advantage of generating numerical basis functions by solving sparse or uniformly banded matrices.

In this communication, the HFEM formulation is modified in such a way that it also results in a sparse or uniformly banded matrix, rather than a partly full and partly sparse nonuniform matrix. The particular formulation used is that first proposed by McDonald and Wexler [2] and thereafter improved and used by others [5]-[9]. The modification is accomplished by changing the sequence of matrix substitutions, a procedure that has been discussed by McDonald and Wexler [6] for general HFEM formulations. The implementation is straightforward, but it substantially improves the computational

Manuscript received September 30, 1987; revised February 3, 1988.

The authors are with the Radiation Laboratory, Department of Electrical Engineering and Computer Science, The University of Michigan, Ann Arbor MI 48109.

IEEE Log Number 8822586. efficiency and enchances the capability of the method. In the following we consider the technique applied to electromagnetic scattering, present sample computations, and compare with other methods.

\section{FORMULATION}

Consider a two-dimensional scattering problem illustrated in Fig. 1 , where the contour $\Gamma$ encloses the scatterer, and the contour $\Gamma_{A}$ is an artificial boundary enclosing $\Gamma$. The details of the HFEM analysis of this problem can be found in [9]; however, for brevity, the notation used here is somewhat different. Application of the finite element analysis to the region enclosed by $\Gamma_{A}$ results in the matrix equation

$$
\left[K_{I A}\right]\left\{\phi_{A}\right\}+\left[K_{I I}\right]\left\{\phi_{I}\right\}=0
$$

where the subscript $A$ denotes the nodes on $\Gamma_{A}, I$ the nodes interior to $\Gamma_{A}$, and $\{\phi\}$ is the discretized unknown field vector. There are no nodes between $\Gamma_{A}$ and $\Gamma$. Equation (1) is referred to as the finite element equation.

To solve (1), another equation is needed to relate $\left\{\phi_{A}\right\}$ and $\left\{\phi_{I}\right\}$ Such an equation can be obtained by using a surface integral equation involving the free-space Green's function, which in discretized form is written as

$$
\left[P_{A A}\right]\left\{\phi_{A}\right\}+\left[P_{A I}\right]\left\{\phi_{I}\right\}=\left\{\phi_{A}^{\text {inc }}\right\}
$$

where $\left\{\phi_{A}^{\mathrm{inc}}\right\}$ is the known incident field vector on boundary $\Gamma_{A}$. Equation (2) is referred to as the equivalent boundary constraint on $\Gamma_{A}$.

The solution for $\left\{\phi_{A}\right\}$ and $\left\{\phi_{l}\right\}$ can then be found by jointly solving (1) and (2). There are two approaches. The first, which is the one commonly used, imposes (2) on (1) and gives the final system equation

$$
\left[K_{n}^{\prime}\right]\left\{\phi_{l}\right\}=\left\{\psi_{I}\right\}
$$

where

$\left[K_{I I}^{\prime}\right]=\left[K_{I I}\right]-\left[K_{I A}\right]\left[P_{A A}\right]^{-1}\left[P_{A I}\right],\left\{\psi_{I}\right\}=-\left[K_{I A}\right]\left[P_{A A}\right]^{-1}\left\{\phi_{A}^{\mathrm{inc}}\right\}$.

In this approach, two matrices have to be solved: one is complex and full matrix $\left[P_{A A}\right]$ having size of $N_{A} \times N_{A}$, and the other is a partly full and partly sparse complex matrix $\left[K_{I I}^{\prime}\right]$ having size of $N_{I} \times N_{I}$, where $N_{A}$ is the total number of nodes on $\Gamma_{A}$ and $N_{I}$ is the total number of nodes interior to $\Gamma_{A}$. Usually, $N_{I}$ is much larger than $N_{A}$, and hence the size of the scatterer to be treated by HFEM is mostly limited by the magnitude of $N_{I}$.

The second approach, which we present here, substitutes (1) into (2) and gives the equation

$$
\left[P_{A A}^{\prime}\right]\left\{\phi_{A}\right\}=\left\{\phi_{A}^{\mathrm{inc}}\right\}
$$

where

$$
\left[P_{A A}^{\prime}\right]=\left[P_{A A}\right]-\left[P_{A I}\right]\left[K_{I I}\right]^{-1}\left[K_{I A}\right]
$$

Mathematically, this second approach is equivalent to the first one; however, computationally it is much more efficient. Here, one also needs to solve two matrices: one is a complex and full matrix $\left[P_{A A}^{\prime}\right]$, but now the other is a symmetric and sparse matrix $\left[K_{I I}\right]$, which becomes real-valued for lossless scatterers and can be narrowly banded if one numbers the nodes properly. A more obvious comparison is given in Table $I$. The difference between the first approach and the second approach is in the properties of the matrices $\left[K_{I I}^{\prime}\right]$ and $\left[K_{I I}\right]$. Solving a symmetric, sparse or uniformly banded 